\title{
Funcionalidad tecnológica del sistema de producción de una granja lechera en la empresa ganadera Valle del Perú
}

\section{Technological functions of the productive system at dairy farm in the cattle breeding enterprise Valle del Perú}

Pedro Pablo Del Pozo R. ${ }^{1}$, PE Fernández ${ }^{1}$, Tania Inés Sanchez G. ${ }^{2}$

${ }^{1}$ Facultad Medicina Veterinaria, Universidad Agraria de La Habana, Cuba. Apartado 18. delpozo@isch.edu.cu / emerio@isch.edu.cu/

${ }^{2}$ Empresa Pecuaria Valle del Perú.

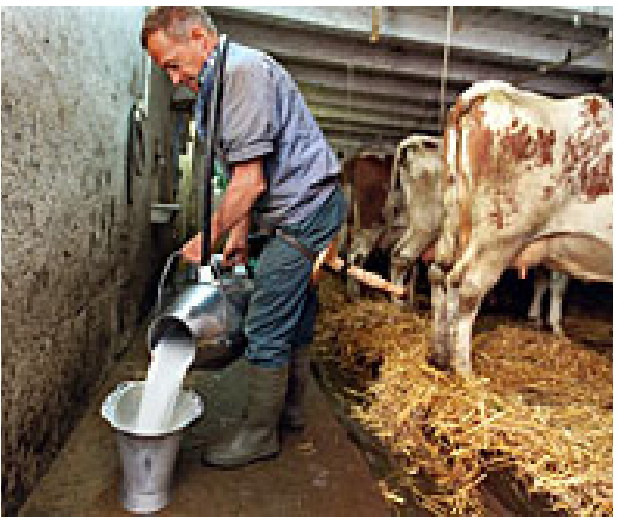

\section{RESUMEN}

Se realizó un diagnóstico con el propósito de determinar la funcionalidad tecnológica y la sustentabilidad de la Granja Zenea de la Empresa Ganadera en la región occidental de Cuba. Se aplicó la metodología de Diagnóstico Rural Rápido (DRP). Las fuentes de información secundarias utilizadas fueron: registros de producción de leche, índices reproductivos y económicos a nivel de granja. La caracterización se efectuó a través de indicadores descriptivos que se relacionan con los componentes del sistema de producción, el productor y sus características, la infraestructura técnico-productiva y aspectos organizacionales. La funcionalidad tecnológica se realizó a partir de variables que se relacionan con los procesos tecnológicos que se identifican con el sistema de producción. Se realizó una jerarquización de problemas y se definieron los principales indicadores que deben servir para medir la sostenibilidad en la propuesta de transformación del sistema. Se analizaron estadísticamente algunas variables productivas y su comportamiento, para los efectos de vaquería, año y bimestre, bajo un modelo lineal de efectos fijos con interacción. El sistema estudiado se caracteriza por ser un sistema de producción típico de la región, con mediano nivel tecnológico y problemas que en su conjunto repercuten en los bajos niveles de producción, ingresos y rentabilidad. Se identificaron a partir de la caracterización realizada los problemas, lo que permitió laborar una estrategia general y específica, así como las principales acciones de carácter técnico-organizativo que se deben tomar para lograr a través del tiempo la sostenibilidad y el mejoramiento de la productividad del sistema estudiado.

Palabras clave: diagnóstico agroecológico, funcionalidad tecnológica, producción de leche.

\section{ABSTRACT}

It was carried out a diagnosis with the purpose of determining the technological functions and sustainability of the Dairy Farm "Zenea" of a cattle raising enterprise in the western region of Cuba. The methodology of Rapid Rural Diagnosis was applied. The secondary sources of information used were: registrations of milk production, reproductive and economic indexes at farm level. The characterization was made through descriptive indicators that are related with the components of the production system, the producer and its characteristics, the technical-productive infrastructure and organizational aspects. The technological functions were determined starting from variables that are related with the technological processes identified with the production system. It was carried out a hierarchical organization of problems and the main indicators that should be appropriate to measure the sustainability were defined in the proposal of transforming the system. Some productive variables and their statistical behavior for the dairy effects, were analyzed every two months and yearly under a lineal model of fixed effects with interaction. The studied system is characterized to be a typical production system of the region, with medium technological level and problems that affect altogether the low production levels, income and profitability. The problems were identified starting from the carried out characterization which allowed working out a general and specific strategy, as well as the main technical and organizational actions that should be taken into account to achieve sustainability and improvement of productivity in the studied system. Key words: Agroecological diagnosis, technological functions, milk production.

Recibido: 9 de diciembre 2013

Aceptado: 20 de marzo 2014 
$\mathrm{E}$ n los últimos años la ganadería cubana tiene como objetivo central incrementar los niveles de productividad y eficiencia económica de sus sistemas ganaderos, y tiene como principal reto satisfacer la creciente demanda de alimentos de la población, más aún cuando se incrementa de forma acelera el valor de los insumos, especialmente los cereales y granos (Iglesias et al., 2005; Funes-Monzote, 2009; Melo, 2012; Iraola, 2013).

En general, los sistemas lecheros del país se encuentran en franca recuperación en sus niveles de eficiencia bioeconómica, pero que aún resultan insuficientes en términos de sus posibilidades potenciales (Guevara et al., 2006; Del Risco, 2007), por lo que se requiere de profundas transformaciones en los sistemas de explotación con base en principios agroecológicos, donde se consideren como ecosistemas y como una simple gestión técnica económica. Esta nueva visión requiere del conocimiento de las leyes que rigen el funcionamiento de cada uno de los elementos y procesos que forman el sistema, con el propósito de maximizar el flujo de energía y el reciclaje de materiales.

Para conocer los problemas reales que enfrentan los productores en la ganadería se han utilizado diferentes metodologías tales como el Diagnóstico Rural Participativo (DRP), lo que le permite a los productores efectuar su propio diagnóstico y auto gestionar su desarrollo, y a través de ello, tomar las decisiones más razonables y asequibles al sistema objeto de estudio (Figueroa y Del Pozo, 2004).

Este trabajo tuvo como objetivo caracterizar la funcionalidad tecnológica y el estado de los recursos en una granja lechera a través de indicadores descriptivos que se relacionan con los componentes del sistema de producción, el productor, la infraestructura técnico productivo y aspectos organizativos, así como determinar la influencia de ellos en la productividad e ingresos de la granja.

\section{MATERIALES Y MÉTODOS}

El trabajo se realizó en una granja lechera de la empresa pecuaria genética Valle del Perú ubicada en el municipio San José de las Lajas, durante los años 2006 y 2007. Para el diagnóstico se empleó la técnica de diagnóstico rural participativo (DRP) descrita por Expósito (2003). Se tuvo en cuenta además las especificidades descritas por Figueroa y Del Pozo (2004); Pérez (2012). Para ello se utilizaron diferentes fuentes de información primaria: encuestas formales a vaqueros, técnicos y directivos de la granja.

Durante el período de estudio la temperatura media anual registró valores de $24.5^{\circ} \mathrm{C}$, con una máxima promedio de $29.8^{\circ} \mathrm{C}$ y mínima promedio de $15.6^{\circ} \mathrm{C}$. Los acumulados anuales de precipitaciones fueron de 1 $600 \mathrm{~mm}$ en régimen estacionario, período lluvioso (mayo-octubre) y período poco lluvioso (noviembre-abril).
Las características fisiográficas del lugar son de plana a ligeramente alomada y poco diseccionada. Además parte del área se encuentra localizada en las alturas cársico-denudativas (zona norte) y las alturas calcáreas sobre margas, areniscas y calizas al sur (Pacheco, 2002).

La caracterización se realizó a través de indicadores descriptivos de las diferentes variables que se relacionan con los componentes del sistema de producción, el productor y sus características, la infraestructura técnico productiva, aspectos organizativos, recursos humanos e inversiones.

Los datos de producción de leche se obtuvieron de los registros del control de producción por unidades y años existentes en la granja. El precio de venta de la leche y su importe final se obtuvieron de las actas de conciliación de la granja con la empresa de lácteos. Los datos de control pecuario, genética y reproducción se obtuvieron a través del programa de SISCOP (Sistema de Control Pecuario) de uso en la entidad productiva.

Se realizaron diferentes análisis de varianzas, utilizando un modelo lineal que consideró como efectos fijos el año $(\mathrm{H})$, el bimestre (S) y vaquerías (V), así como las interacciones entre los efectos considerados, en las diferentes variables estudiadas.

Así el modelo resultó: $\mathrm{Y}_{\mathrm{ijkl}}=\mathrm{M}_{\mathrm{o}}+\mathrm{H}_{\mathrm{i}}+\mathrm{S}_{\mathrm{j}}+\mathrm{V}_{\mathrm{k}}+(\mathrm{HxSxV})_{\mathrm{ijk}}+\mathrm{e}_{\mathrm{ijk} \mathrm{k}}$

Donde:

$$
\begin{aligned}
& \mathrm{Y}_{\mathrm{ijkl}}=\text { Variable en estudio } \\
& \mathrm{M}_{\mathrm{o}}=\text { Media general } \\
& \mathrm{H}_{\mathrm{i}}=\text { Efecto de la k- ésimo bimestre, } \mathrm{k}=1,2, \ldots \mathrm{n} \\
& \mathrm{S}_{\mathrm{j}}=\text { Efecto de j-ésimo año, } \mathrm{j}=1,2, \ldots . \mathrm{n} \\
& \mathrm{V}_{\mathrm{k}}=\text { Efecto de la i-ésima vaquería, } \mathrm{i}=1,2 \ldots \mathrm{n} \\
& \mathrm{HxSx}_{\mathrm{ijk}}=\text { interacción bimestre }- \text { año }- \text { vaquería } \\
& \mathrm{e}_{\mathrm{ijkl}}=\text { error aleatorio }
\end{aligned}
$$

Para el procesamiento de los datos se empleó el software estadístico STATGRAPHICS 5.1 y en los casos necesarios, se empleó la dócima de rango múltiple de Duncan (1955) para la comparación de las medias.

\section{RESULTADOS Y DISCUSIÓN}

El suelo predominante en el ecosistema estudiado es el Pardo Carbonatado, con categoría agroproductiva IV (Hernández et al., 2005) el que ocupa más del 95\% del área de las unidades contempladas en el estudio.

La granja posee una extensión de 1015.03 hectáreas, con un área agrícola total de 974.58 hectáreas; de las cuales el $73 \%$ está cubierto de pastos cultivados y el $13.14 \%$ de forrajes; el resto lo constituyen plantas arvenses y arbustivas. 
La producción de leche es el renglón fundamental de la granja y sus valores durante los años 2006-2007 se muestran en el cuadro 1.

En los análisis estadísticos realizados se encontró que el año fue significativo para las diferentes variables estudiadas, con excepción de vacas en ordeño y \% de vacas en ordeño (cuadro 1), lo cual pudiera estar relacionado con el deterioro de los indicadores reproductivos, reflejados en la tasa de natalidad (76.4\%), animales que paren y no entran al ordeño, casos donde se acortan las lactancias, por inestabilidad de la alimentación o por deficiencias en el manejo del rebaño.

El efecto vaquería fue significativo para todas las variables, aspecto que denota diferencias en el manejo empleado en los rebaños de cada unidad productiva, lo cual corrobora que el manejo animal constituye un elemento clave en el funcionamiento de los sistemas de producción animal (García, 2005)

El bimestre del año fue significativo en todos los indicadores, excepto en la variable masa total de vacas por unidad pecuaria, lo cual se corresponde con los cambios estacionales en la producción de forrajeras en la granja. Los bimestres más favorables para la producción de leche por vaca y total, productividad por área e ingresos ocurrieron durante los meses del período lluvioso.
Estas diferencias por época, demuestran las características estacionales en la producción de leche, lo que pudiera ser consecuencia de no garantía de la oferta sostenida de pastos y forrajes en el período poco lluvioso, para que se minimicen las brechas productivas a lo largo de todo el año (Martín, 2004; Martínez et al., 2010). Por tanto, estos sistemas se encuentran sujetos a los efectos de los diferentes elementos climáticos, lo que indica la necesidad de poder ajustar más las tecnologías a las condiciones concretas de la unidad productiva, mediante una cultura rural más adecuada al productor, con conocimientos y habilidades necesarias, por la vía de la capacitación e intercambios de experiencias (Senra, 2011; Senra, 2005).

La vacas totales no mostraron variación significativa, lo que indica que para esta categoría zootécnica la masa animal se mantuvo estable en la granja en las diferentes épocas del año, aspecto muy importante para lograr una estabilidad en la masa en las unidades lecheras (Melo, 2012; Loyola, 2010).

Dado que las interacciones no son significativas en ningún de los casos, puede asegurarse independencia entre los efectos incluidos en el modelo. La alta significación encontrada en los análisis estadísticos, nos indica la confiabilidad de los análisis realizados y sus resultados.

Cuadro 1. Efecto del año, vaquería y bimestre sobre algunos de los indicadores relacionados con la producción de leche, vacas en ordeño, carga animal e ingresos en la granja

\begin{tabular}{|c|c|c|c|c|c|c|c|c|c|c|}
\hline \multicolumn{11}{|c|}{ Variables } \\
\hline Años & $\begin{array}{l}\mathrm{kg} / \mathrm{vaca} \text { en } \\
\text { ordeño }\end{array}$ & $\begin{array}{c}\text { Vacas en } \\
\text { ordeño }\end{array}$ & $\begin{array}{c}\text { Carga animal } \\
\text { vacas/ha }\end{array}$ & $\begin{array}{l}\% \text { vacas } \\
\text { en ordeño }\end{array}$ & Vacas & & $\begin{array}{r}\text { Precio } \\
\text { totale }\end{array}$ & & $\begin{array}{c}\text { Ingresos } \\
\text { (\$) }\end{array}$ & \\
\hline 2006 & 6.45 & 47.3 & 1.31 & 61.00 & 76.90 & & 0.98 & & 9051.24 & \\
\hline 2007 & 5.92 & 46.7 & 1.35 & 60.80 & 75.00 & & 1.70 & & 13849.9 & \\
\hline E.E+- & 0.12 & 0.65 & 0.01 & 0.59 & 0.52 & & 0.04 & & 381.63 & \\
\hline Sign. & $* *$ & NS & $* *$ & NS & * & & $* * *$ & & $* * *$ & \\
\hline \multicolumn{11}{|c|}{ Vaquerías } \\
\hline 1 & $6.91 \mathrm{c}$ & 58.4 c & $1.94 \mathrm{~d}$ & $64.6 \mathrm{~cd}$ & 90.4 & $\mathrm{e}$ & 1.47 & $\mathrm{c}$ & 17199.5 & $\mathrm{~d}$ \\
\hline 2 & $5.93 \mathrm{~b}$ & $64.9 \mathrm{~d}$ & $1.25 \mathrm{ab}$ & $66.0 \mathrm{~d}$ & 98.4 & $\mathrm{f}$ & 1.37 & $\mathrm{bc}$ & 15774.9 & d \\
\hline 3 & $7.10 \mathrm{c}$ & 37.4 a & $1.21 \mathrm{ab}$ & $57.0 \mathrm{a}$ & 66.9 & b & 1.33 & $a b$ & 9983.9 & $\mathrm{bc}$ \\
\hline 4 & $6.86 \mathrm{c}$ & $45.9 \mathrm{~b}$ & $1.23 \mathrm{ab}$ & 62.7 bcd & 73.2 & $\mathrm{c}$ & 1.25 & $\mathrm{a}$ & 11564.3 & $\mathrm{c}$ \\
\hline 5 & $6.12 \mathrm{~b}$ & $43.6 \mathrm{~b}$ & $1.34 \mathrm{c}$ & $61.5 \mathrm{abcd}$ & 70.8 & $\mathrm{c}$ & 1.37 & $\mathrm{bc}$ & 10553.2 & $\mathrm{bc}$ \\
\hline 6 & $4.89 \mathrm{a}$ & $43.7 \mathrm{~b}$ & $1.18 \mathrm{a}$ & 60.9 abcd & 71.4 & $\mathrm{c}$ & 1.31 & $a b$ & 9004.4 & $a b$ \\
\hline 7 & $6.00 \mathrm{~b}$ & $46.1 \mathrm{~b}$ & $1.29 \mathrm{bc}$ & $59.0 \mathrm{abc}$ & 77.9 & d & 1.32 & $a b$ & 10119.5 & $\mathrm{bc}$ \\
\hline 8 & $5.69 \mathrm{~b}$ & $33.5 \mathrm{a}$ & $1.19 \mathrm{a}$ & $56.8 \mathrm{ab}$ & 58.6 & $\mathrm{a}$ & 1.30 & $\mathrm{ab}$ & 7405.05 & $\mathrm{a}$ \\
\hline E.E+- & 0.20 & 1.81 & 0.03 & 1.87 & 1.17 & & 0.04 & & 629.37 & \\
\hline Sign. & $* * *$ & $* * *$ & $* * *$ & $* *$ & $* * *$ & & $*$ & & $* * *$ & \\
\hline \multicolumn{11}{|c|}{ Bimestres } \\
\hline 1 & $5.25 \mathrm{a}$ & 48.1 & $1.31 \mathrm{ab}$ & 63.8 & 75.0 & & 1.0 & $\mathrm{a}$ & 7710.61 & $\mathrm{a}$ \\
\hline 2 & $5.38 \mathrm{ab}$ & $48.8 \mathrm{~b}$ & $1.29 \mathrm{a}$ & $63.6 \mathrm{c}$ & 76.2 & & 0.96 & $\mathrm{a}$ & 7760.14 & $\mathrm{a}$ \\
\hline 3 & $5.94 \mathrm{~b}$ & $48.0 \mathrm{~b}$ & $1.27 \mathrm{a}$ & $62.3 \mathrm{bc}$ & 76.3 & & 0.96 & $\mathrm{a}$ & 8472.01 & a \\
\hline 4 & $7.52 \mathrm{c}$ & $44.9 \mathrm{a}$ & $1.34 \mathrm{bc}$ & 57.4 a & 77.1 & & 1.72 & $\mathrm{~b}$ & 16855.0 & d \\
\hline 5 & $7.06 \mathrm{c}$ & $44.4 \mathrm{a}$ & $1.39 \mathrm{~d}$ & 58.3 a & 75.2 & & 1.74 & b & 15003.4 & $\mathrm{c}$ \\
\hline 6 & $5.99 \mathrm{~b}$ & $46.2 \mathrm{ab}$ & $1.37 \mathrm{~cd}$ & $60.1 \mathrm{ab}$ & 75.9 & & 1.66 & $\mathrm{~b}$ & 12902.3 & $\mathrm{~b}$ \\
\hline E.E+- & 0.22 & 0.98 & 0.01 & 1.03 & 0.89 & & 0.07 & & 661.01 & \\
\hline Sign. & $* * *$ & $* *$ & $* * *$ & $* * *$ & N.S & & $* * *$ & & $* * *$ & \\
\hline
\end{tabular}

$\mathrm{a}, \mathrm{b}, \mathrm{c}, \mathrm{d}$, Valores con letras no comunes por columna difieren a $p<0.05$ (Duncan, 1955). 


\section{PRODUCCIOONAWMMAL}

En el cuadro 2 y 3 se muestran el estado reproductivo del rebaño durante los dos años de estudio. El porciento de gestación en los años estudiados ha estado muy por debajo del $50 \%$, lo que se considera un signo de alarma para un rebaño en producción (Soto, 2010; Sixto, 2000). Esta situación puede estar relacionada al gran número de vacas repetidoras que existen en el rebaño, asociado al mal celaje y al estado físico con que quedan las vacas al parir. Toda esta situación trae consigo que se pierdan celos o que no se insemine en el momento óptimo. Situación semejante presenta el intervalo parto primer servicio e intervalo parto - parto.
Cuadro 2. Estado reproductivo del rebaño durante el período estudiado

\begin{tabular}{cccccc}
\hline Año & \multicolumn{5}{c}{ Indicadores } \\
\cline { 2 - 6 } & HBP & Gestantes (\%) & Inseminadas (\%) & Recentinas (\%) & Vacías (\%) \\
\hline 2006 & 822 & 31.90 & 44.10 & 12.70 & 11.30 \\
2007 & 807 & 31.80 & 48.17 & 12.25 & 7.78 \\
\hline
\end{tabular}

HBP: Hembras bajo plan de reproducción.

Cuadro 3. Comportamiento de algunos indicadores reproductivos del rebaño

\begin{tabular}{ccccc} 
Año & \multicolumn{4}{c}{ Indicadores } \\
\cline { 2 - 5 } & $\begin{array}{c}\text { Natalidad } \\
(\%)\end{array}$ & $\begin{array}{c}\text { Intervalo parto } \\
\text { parto (día) }\end{array}$ & $\begin{array}{c}\text { Intervalo parto } \\
1^{\mathrm{er}} \text { servicio (día) }\end{array}$ & $\begin{array}{c}\text { Servicio por } \\
\text { gestación }\left(\mathrm{N}^{\circ}\right)\end{array}$ \\
\hline 2006 & 74.10 & 494.4 & 102.40 & 2.90 \\
2007 & 78.70 & 478.0 & 91.40 & 3.00 \\
\hline
\end{tabular}

En el cuadro 4 se muestra de forma resumida los resultados del diagnóstico agroecológico realizado, lo cual nos permitió identificar los problemas que afectan el proceso de produc-

ción en la granja y su funcionalidad tecnológica, estableciendo cuales eran los de mayor importancia y la orientado posible de soluciones (figura 1).

Cuadro 4. Caracterización agroecológica del sistema estudiado

\begin{tabular}{|c|c|c|}
\hline Limitantes & Causas & Efectos \\
\hline $\begin{array}{l}\text { Reducida diversidad } \\
\text { productiva }\end{array}$ & $\begin{array}{l}\text { Dependencia por mantener la especiali- } \\
\text { zación producción lechera }\end{array}$ & $\begin{array}{l}\text { Tendencia a la simplificación estructural y } \\
\text { funcional del agroecosistema }\end{array}$ \\
\hline \multirow[t]{2}{*}{$\begin{array}{l}\text { Limitada diversidad } \\
\text { de especies }\end{array}$} & $\begin{array}{l}\text { Manejo de monocultivo de especies fo- } \\
\text { rrajeras }\end{array}$ & \multirow[t]{2}{*}{$\begin{array}{l}\text { Pérdida de la biodiversidad en el ecosistema } \\
\text { pastizal }\end{array}$} \\
\hline & $\begin{array}{l}\text { Pobre tendencia a la integración ganade- } \\
\text { ría- agricultura. }\end{array}$ & \\
\hline \multirow{4}{*}{$\begin{array}{l}\text { Manejo inadecuado } \\
\text { del pastoreo }\end{array}$} & Limitada rotación del pastoreo. & Deterioro progresivo de los pastizales. \\
\hline & \multirow{3}{*}{$\begin{array}{l}\text { No control del tiempo de estancia de los } \\
\text { animales en potreros. }\end{array}$} & Incremento en la densidad de malezas. \\
\hline & & Compactación de los suelos. \\
\hline & & Mal manejo y aprovechamiento de los pastos. \\
\hline \multirow{2}{*}{$\begin{array}{l}\text { Bajos índices pro- } \\
\text { ductivos }\end{array}$} & \multirow{2}{*}{$\begin{array}{l}\text { Baja disponibilidad en cantidad y calidad } \\
\text { de los pastos. }\end{array}$} & Producción ganadera no sostenida. \\
\hline & & Reducción de ingresos para la familia. \\
\hline \multirow{2}{*}{$\begin{array}{l}\text { Escaso uso de técni- } \\
\text { cas alternativas }\end{array}$} & \multirow{2}{*}{$\begin{array}{l}\text { Escasas opciones para la suplementación } \\
\text { alimentaria. }\end{array}$} & Alta dependencia de los insumos. \\
\hline & & No aprovechamiento de los recursos locales. \\
\hline \multirow{3}{*}{$\begin{array}{l}\text { Bajo porciento de } \\
\text { gestación y partos }\end{array}$} & Problemas en la detección del celo. & \multirow{3}{*}{$\begin{array}{l}\text { Deteriores de los índices reproductivos y en } \\
\text { particular la natalidad. }\end{array}$} \\
\hline & Inseminación en momento óptimo. & \\
\hline & Baja condición corporal. & \\
\hline
\end{tabular}




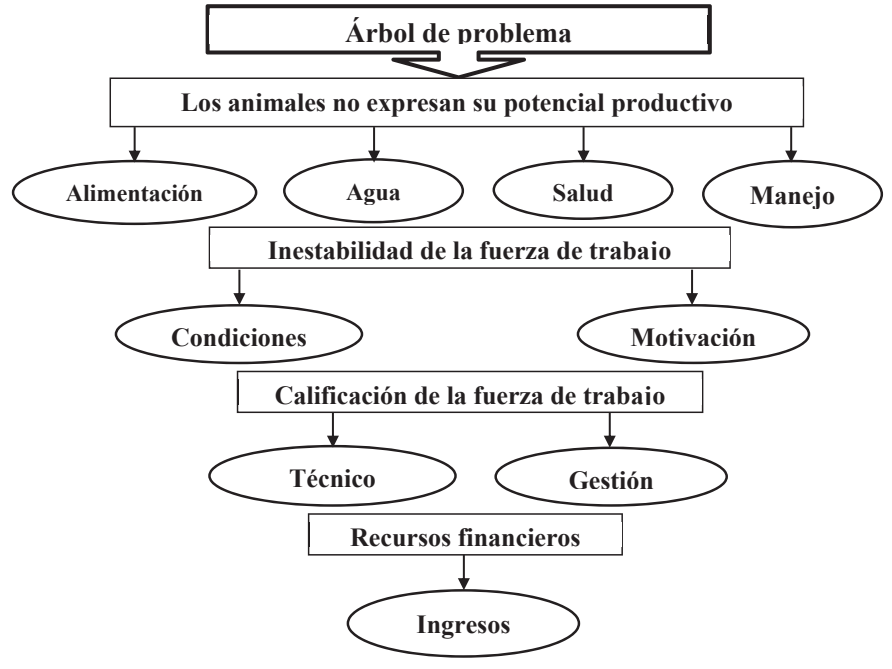

Figura 1. Principales problemas que limitan el potencial productivo y la funcionalidad tecnológica en la granja.

\section{CONCLUSIONES}

El sistema estudiado se caracteriza por ser un sistema de producción típico de la región, con mediano nivel tecnológico, con diferentes problemas que en su conjunto repercuten en los bajos niveles de producción, ingresos y rentabilidad en la granja.

Se identificaron, a partir de la caracterización realizada, los problemas de mayor importancia y se recomienda prestar especial atención al problema de degradación presente en el ecosistema, asícomolograrun aprovechamientoracionaldelos recursos forrajeros locales, la diversificación de la producción, el reciclaje de productos y el uso mínimo de recursos externos.

\section{REFERENCIAS BIBLIOGRÁFICAS}

Del Risco, GS; Guevara, R; Guevara, G; Curbelo, L; Soto, S. 2007. Evaluación del comportamiento productivo de vaquerías comerciales en relación con el patrón de pariciones anuales. I. Análisis comparativo de la eficiencia de los patrones. Revista de Producción Animal 19 (1):13-19.

Duncan, DB. 1955. Multiple range and multiple F tests. Biometrics 11:1-12.

Expósito V, M. 2003. Diagnóstico rural participativo. Guía práctica. Proyecto comunicación y didáctica. Centro Cultural Poveda. República Dominicana. p. 118.

Figueroa Felipe Y; Del Pozo, PP. 2004. Diagnóstico rural participativo de una finca ganadera. Memorias Simposio Internacional de ganadería agroecológica. Instituto de investigaciones de pastos y forrajes (IIPF)/ MINAGRIC. Celebrado Nov-2004, Holguín, Cuba.

Funes Monzote, F. 2009. Agricultura con futuro. La alternativa agroecológica para Cuba. Estación Experimental "Indio Hatuey", Universidad de Matanzas. $176 \mathrm{p}$.

García López, R. 2005. Aspectos de la nutrición bovina de interés práctico que puede influir en su eficiencia. Conferencia del curso "producción intensiva de leche". ICA. La Habana. CU Nov. 30 p.

Guevara, R; Guevara, G; Sánchez, M; Curbelo, L; Véliz; MC; Pedraza, R; Villareal, O. 2006. El contexto socioeconómico global y regional y sus efectos sobre la producción ganadera (artículo reseña primera parte). Hidra. Boletín informativo para ganaderos. CEDEPA N ${ }^{\circ} 18$, p. 1 .

Hernández, A, Ascanio, MO; Morales, M; Cabrera, A. 2005. Correlación de la nueva versión de la clasificación genética de los suelos de Cuba con las clasificaciones internacionales y nacionales: una herramienta útil para la investigación, docencia y producción agropecuaria. Ed. Instituto Nacional de Ciencias Agrícolas. La Habana, CU. 62 p.

Iglesias, J; Fernando, R; Funes-Monzote, HM; Mildrey, S. 2005. Los sistemas de producción agropecuaria como alternativa agroecológica de producción en las condiciones actuales. In memorias del III evento de actualización de medicina veterinaria, zootecnia e hidrobiológicos, Guatemala. 17-19 junio 2005.

Iraola, J. 2013. Rediseño y manejo de un arreglo silvopastoril para mejorar la capacidad de carga biológica con ganado de engorde. Tesis en opción al grado de Doctor en Ciencias Veterinarias. ICA- UNAH, La Habana, CU. 119 p.

Loyola O, CJ. 2010. Efectos del periodo de ocurrencia y la intensificación de la parición al inicio de la época lluviosa, sobre la eficiencia bioeconómica de vaquerías comerciales. Tesis en opción al grado de Doctor en Ciencias Veterinarias. Universidad de Camagüey, Camagüey, CU. 144 p.

Martín, PC. 2004. La alimentación del ganado con caña de azúcar y sus subproductos. Editorial del Instituto de Ciencia Animal. La Habana, CU. 193 p.

Martínez, RO; Aguilar, PI; Torres, V. 2010. Análisis del impacto de la tecnología de los bancos de biomasa con el pasto Cuba CT-115 en nueve lecherías de la UBPC desembarco del Granma, Villa Clara, CU. III Congreso reproducción Animal Tropical. Palacio de Convenciones, La Habana, CU. p 112-115. 
Melo, MJ. 2012. Bases para el reordenamiento de los sistemas lecheros cooperativos en la provincia de Ciego de Ávila. Tesis en opción al grado de Doctor en Ciencias Veterinarias. UNICA, Ciego de Ávila, CU. 147 p.

Pacheco, MA. 2002. Análisis y diferenciación especial de indicadores socioeconómicos de la Empresa Pecuaria Genética "Valle del Perú”. Tesis para optar por el grado de Máster en Ciencias Geográficas. Universidad de La Habana. Ciudad Habana. CU. 89 p.

Pérez, YA. 2012. Modelación Estadístico-Matemática con variables mixtas para el estudio de la sostenibilidad social en una empresa ganadera bovina. Tesis en opción al grado de Doctor en Ciencias Veterinarias. Universidad Agraria de la Habana, Mayabeque, CU. 135 p.

Senra, A. 2011. Cultura de trabajo para garantizar la sostenibilidad; eficiencia e impacto final de las tecnologías. Avances en investigación agropecuaria (AIA). 15(2):3-12

Senra, A. 2005. Índices para controlar la eficiencia y sostenibilidad del ecosistema del pastizal en la explotación bovina. Revista Cubana de Ciencia Agrícola. 39(1):13-21.

Sixto, G. 2000. Soluciones de problemas reproductivos en la vaca. Disponible en soporte magnético. Unidad Docente Nazareno. Facultad de Medicina Veterinaria. UNAH. La Habana. CU. p 94-96.

Soto S, SA. 2010. Influencia de la distribución y concentración de la parición en la eficiencia bioeconómica de la producción de leche en vaquerías del municipio de Jimaguayú, Camagüey. Tesis en opción al grado de Doctor en Ciencias Veterinarias. Universidad de Camagüey, Camagüey, CU. 139 p. 